\title{
APERTURA Y DESARROLLO REGIONAL EN LA PERIFERIA CAPITALISTA: EL CASO DE MÉXICO
}

\author{
José A. Alonso*
}

\section{INTRODUCCIÓN}

Las políticas socio-económicas iniciadas en la década de los ochenta en México presentan una clara orientación aperturista. Hoy día es un lugar común asociar esa tendencia con la estrategia neoliberal'. Desde el comienzo de esa década, atentos observadores de la escena nacional ya habían anunciado las características que tal modelo asumiría, si es que llegara a implantarse en nuestro país. (Entre otros: Cordera y Tello, 1981: 79 - 106). La esencia de tal proyecto, según Cordera y Tello, es que se trata de un proyecto metropolitano. En otras palabras, es una estrategia diseñada por las grandes potencias, en nuestro caso por los Estados Unidos, para reestructurar el sistema capitalista internacional en función de sus intereses financieros y transnacionales. (SaxeFernández, 1992: 135 - 179).

En esta propuesta del gran capital norteamericano se percibe ya el germen de lo que llegaría a ser el Tratado de Libre Comercio para Norte América (en adelante, TLCNA). Aunque lo cierto es que los hechos sobrepasarían con amplitud la aguda visión de Cordera y Tello. Ambos autores indicaron que la culminación de la visión neoliberal sería una creciente integración de México a la economía yanqui gracias a la armonización de la política del Estado mexicano con la de los Estados Unidos (ib.:8l) ${ }^{2}$.

Lo que Tello y Cordera no podían ni sospechar en aquel entonces era el grado de sometimiento a los intereses del país del norte al que llegarían las élites políticas y financieras mexicanas. En efecto, tanto los líderes norteamericanos -cosa fácil de entender-, como los jerarcas mexicanos parten de aceptar "como proposición maestra la de que los intereses a largo plazo de las sociedades norteamericana y mexicana pueden ser mejor satisfechos si ambas economías se abocan conscientemente a la construcción de un sistema global de mutua complementación" (ib.:81).

En el contexto de estas supuestas mutuas conveniencias, los gobiernos de ambos países se dedicaron en los años siguientes a dar forma legal a tan asimétrica convergencia en el pacto hoy conocido como el TLCAN ${ }^{3}$. Por lo demás, es conveniente señalar desde ahora que la inclusión de Canadá en el TLC no atempera ni por su posición geográfica, ni por su poderío económico la relación de dependencia de México con respecto a los EE.UU. (Gutiérrez Haces, 1992: 66 - 71).

\section{ESTADOS UNIDOS: PAÍS MÁS BENEFICIADO POR EL TLC}

Antes de entrar en el análisis, de lo ocurrido en los estados de Haxcala y Aguascalientes a partir de 1994, es conveniente dejar bien claro qué país ha sido el más beneficiado hasta ahora por el TLC.

El liderazgo y predominio de los EE.UU. en "todas las Américas" son reconocidos por todos los observadores internacionales, incluidos autores franceses tan conocidos como (Fossaert: 1991, 476). No obstante, este mismo autor afirma (ib.:452) que el libre intercambio continental no será una panacea para los EE.UU. La zona comercial que se extienda desde Yucatán hasta la península del Labrador, apoyada por las políticas públicas neoliberales del gobierno Norteamericano, provocará un reforzamiento de su poderío, pero a costa de sacrificar sus políticas de bienestar social y de frenar la carrera de los armamentos.

Sin embargo, no se debe olvidar que México es parte de Norteamérica, no simplemente de las Américas. La cercanía

Universidad de Las Américas - Puebla. UDLA-P.

1. La bibliografía sobre el neoliberalismo es abundante. Para obtener una visión crítica puede consultarse: Varios autores (1990): América Latina: Critica del Neoliberalismo. México, D.F.: Centro de Estudios para un Proyecto Nacional Alternativo.

2. Este proceso de integración asume hoy día características tan atípicas que ya comienza a hablarse del surgimiento de una cuarta nación en Norteamérica. Puede verse al respecto: Brown, Timothy C. (1997): "The Fourth Member of NAFTA: The U.S. - Mexico Border”. En The Annals Of The American Academy Of Political And Social Science, vol. 550: 105 . 129 (march).

3. Una muestra excelente de la activa preparación del proceso que en la firma del TLCAN es el libro Cambio Estructural En México y en El Mundo. (Fondo de Cultura Económica, México, 1987), en el que aparecen contribuciones tan significativas como las de Miguel de la Madrid -en aquel momento presidente de la República Mexicana-y de su sucesor, el hoy errante Carlos Salinas de Gortari, pero que en aquel momento fungía como jefe de la efímera Secretaría de Programación y Presupuesto. 
geográfica y los intereses comerciales (petróleo y minerales, entre otros) de los EE.UU. convierten a México en un socio prioritario. De ahí que el TLC no pueda considerarse sólo como un proceso ambicioso e importante a nivel americano. El TLCNA, subraya Jordi Vilaseca, es "una asociación sustancialmente distinta a las de iniciativa latinoamericana, debido a que es el resultado de la carrera hacia la hegemonía en la creación de un único mercado mundial" (Vilaseca y Requena, 1995: 445). En otras palabras, el TLCAN representa para los EE.UU. sólo un jalón para conquistar -o reconquistar- el liderazgo indiscutible del comercio mundial.

Este carácter con nítidos ribetes yanquis del TLCNA ha sido muy tenido en cuenta, por ejemplo, por las Cumbres Iberoamericanas que en sus documentos finales mencionan la necesidad de proseguir con la estrategia de la integración, pero no citan en ningún momento al TLCNA (ib.:445) ${ }^{4}$.

Por lo demás, esta orientación benéfica del TLCNA con relación a los EE.UU. no dimana primordialmente de políticas coyunturales, como las promovidas por Ronald Reagan a principios de los ochentas. Las mayores ventajas caerán siempre en el lado norteamericano porque la actual división internacional del trabajo y las ventajas comparativas, que de ahí dimanan, para los Países Capitalistas Centrales determinan con ineludible necesidad que el comercio exterior tienda a reforzar las desigualdades existentes entre el Centro y la Periferia (Talavera Déniz y Martínez Peinado, 1995: 365).

Añadamos, por lo demás, que esta añeja tendencia estructural del sistema-mundo capitalista se ha visto incrementada en los años ochenta y que, además, afecta frontalmente a México. Primero, por el notable aumento del proteccionismo, concretamente en los EE.UU. durante los últimos veinticinco años, el cual se enfoca directamente hacia México después del Japón (ARRIOLA: 1997). En segundo lugar, otro factor que afectó con todo rigor a México fue la modificación del tipo de interés de la deuda externa de los países periféricos. Como es sabido, la crisis provocada por la deuda externa explotó en agosto de 1982 cuando México se declaró insolvente. Pero como el tema de la deuda es bastante conocido y estudiado (Estay, 1996), sólo señalaremos dos aspectos muy relacionados con las próximas secciones de esta ponencia. En primer lugar, el incremento reciente de la deuda externa mexicana se explica en gran parte por las mayores necesidades importadoras existentes en los procesos de producción (ibi.:146). Este aspecto está íntimamente relacionado con el segundo, es decir, con "el papel desempeñado, durante el periodo de rápido endeudamiento, por las filiales de empresas trasnacionales situadas en América Latina -y, por tanto, en México- (ib.:152).

Estay Reyno subraya que las filiales de las empresas trasnacionales contribuyen en forma significativa al déficit de la región puesto que exportan menos o igual e importan más que las empresas nacionales ; además, aquí desempeña "un papel importante el elevado monto del comercio intrafirma y las consiguientes prácticas de sobrefacturación de importaciones, subfacturación de exportaciones y, en general, de establecimiento de precios de transferencia entre las filiales y sus matrices" 5 .

¿Cúal fue la situación en los años noventa, es decir, en el periodo previo a la entrada en vigor del TLCNA? El aspec- to más destacable, por su incidencia en la estructura industrial de México, es que durante el sexenio de Carlos Salinas de Gortari "un porcentaje cada vez mayor de los capitales extranjeros ingresó a la economía mexicana no para producir, sino para dedicarse a la especulación, atraídos por las elevadas tasas de interés... y en condiciones de emprender rápidamente la retirada si cambiaban sus expectativas" (Estay: 239). Una primera manifestación de los riesgos inherentes a tal situación financiera la experimentó México en diciembre de 1994. Sobra añadir que esta creciente vulnerabilidad de la economía mexicana no ha disminuido con la entrada en vigor del TLCNA a partir del 1" de enero de 1994. Por el contrario, la última debacle económica tuvo lugar en diciembre de ese mismo año.

\section{LAS REGIONES DE MÉXICO ANTE EL TLCNA: UNA PROPUESTA METODOLÓGICA}

¿Cúales han sido las consecuencias de este "salto en el vacío" dado por México al firmar el TLC en su versión actual? ¿Qué beneficios han obtenido México como país con este tratado y, más específicamente, su sector manufacturero? ¿Qué cambios ha experimentado la industria mexicana desde que se consolidó la apertura comercial iniciada a partir de 1982 ?

El éxito experimentado por las ET norteamericanas en sus incursiones en territorio mexicano es un fiel indicador de la desestructuración sufrida por la industria nacional durante el mismo periodo. Alguien podría pensar que "desestructurar" la industria tradicional es un paso firme hacia la modernidad, pero el caso de Tlaxcala nos mostrará que el avance hacia una sociedad más autónoma y, en este sentido, más moderna no viene de la mano de la simple renovación tecnológica y menos cuando ésta viene impuesta desde el extranjero.

Existe un complejo cúmulo de factores que es preciso tener en cuenta si pretendemos impulsar en México un desarrollo socio-económico independiente y autónomo (no autárquico). Recientemente se ha insistido en México en la necesidad de revitalizar el "federalismo", como la única manera de asegurar un desarrollo más equitativo entre las regiones del país (Diaz Cayeros, 1995: 9). Desde luego no es posible negar la incidencia de las variables políticas para encontrar la solución adecuada, pero allí mismo se reconoce que la apertura comercial mexicana -consagrada en el TLCNA, añadimos-no ha venido acompañada por la vigorización de un federalismo más equilibrado ${ }^{6}$.

4. Esta misma tendencia proyanqui de los acuerdos impulsados en América Latina por los EE.UU. se profundizó aún más a fines de 1994 cuando, según los mismos autores, "los EE.UU. convocaron la Cumbre del Hemisferio en Miami, cuyo eje principal fue la ampliación del TLCAN a nuevos miembros (incorporando a Chile), lo que se configura como Una estrategia radicalmente diferente a la apuntada por las Cumbres Iberoamericanas" (énfasis a-adido;ib. p.445).

5. Sobre el tema de las empresas matrices norteamericanas y sus filiales en México puede verse el artículo del Isidro Morales (1996): "Hierarchical and arms length markets under NAFTA. The case of Mexican petrochemical and textile industries" Versión mimeografiada.

6. En este sentido, el autor reconoce expresamente la diferencia notable entre la apertura comercial de México y la liberalización lograda por 
Sin olvidar la importancia del aspecto político, creemos que se requiere un planteamiento más fundamental y comprensivo porque el proceso de industrialización seguido durante los últimos tres lustros en México ha producido un profundo desequilibrio regional (Delgadillo Macías: 1993, 11). Este enfoque debe incluir, en primer término, el uso y la distribución del espacio, tanto en el nivel internacional como en el intranacional.

La creciente internacionalización del capital, provocada ante todo bajo el liderazgo de las ET (empresas transnacionales), ha generado una nueva fase en las relaciones entre el centro y la periferia capitalistas. Este es un primer aspecto básico en la actual división del espacio a nivel mundial. Dada la naturaleza colonizadora del capital, inscrita en el mismo proceso de acumulación ampliada, nos interesa diagnosticar ahora las nuevas funciones que se asignan al espacio primero en el nivel internacional. A partir del planteamiento del sistema-mundo capitalista, desarrollado por Immanuel Wallerstein (Wallerstein, 1979), nuestra primera tarea consistiría en especificar cómo se utiliza el espacio de la periferia capitalista como factor de producción.

Siguiendo el análisis esbozado por Javier Delgadillo (ib.: 119), cabe reconocer que el nuevo patrón de industrialización surge en México a partir de 1994 mediante la yuxtaposición de las empresas tradicionales mayoritarias -en supuesto proceso de modernización-y las nuevas empresas maquiladoras de punta que llegan en nuevas oleadas ${ }^{7}$.

La segunda tarea metodológica presupone la primera y la especifica a nivel regional, porque el impacto de las ET en la periferia capitalista no es uniforme, sino que presenta profundas diferencias regionales. En el esquema usado por Javier Delgadillo, la intensa internacionalización del capital se apoya en nuevas modalidades de inversión, tanto extranjera como nacional, las cuales generan modalidades productivas nuevas $y$, por ende, un nuevo ordenamiento territorial

\section{AGUASCALIENTES Y TLAXCALA: DOS IMPAC- TOS REGIONALES DE LA GLOBALIZACIÓN}

En México, los diminutos estados de Aguascalientes y Tlaxcala nos ofrecen dos magníficos ejemplos del impacto diferencial generado por las incursiones del capital transnacional en dos regiones del altiplano mexicano ${ }^{8}$. Estos dos casos regionales presentan notables coincidencias y divergencias, al mismo tiempo, en el desarrollo socio-económico experimentado durante los últimos años.

Los estados de Aguascalientes y de Tlaxcala ofrecen una serie de "ventajas" similares que actúan como imanes para el capital transnacional. Entre éstas destacan:

a) Los dos estados poseen una fuerte tradición artesanal y manufacturera, específicamente en el área de la industria del vestido. Gracias a esta experiencia secular, la fuerza de trabajo en ambas regiones puede incorporarse casi de inmediato a las nuevas tareas exigidas por la globalización industrial, a pesar de las altas exigencias del mercado norteamericano. Además, en los dos estados se ha dado en los últimos años una evidente reducción de esfuerzos guberna- mentales para reanimar a las industrias tradicionales. De ahí que, como veremos en el caso de Tlaxcala, sea más atractivo para las pequeñas y medianas empresas el incorporarse a las nuevas redes de la maquila internacional que tratar de crecer de manera independiente.

b) Los dos estados están localizados en la zona central del país y cruzados por importantes vías de comunicación, que incluyen carreteras y ferrocarriles. Este rasgo es uno de los aspectos decisivo, por otra parte, para atraer al capital internacional hacia estos pequeños estados de la República Mexicana.

c) En ambos estados el apoyo estatal hacia el sector industrial se ha dirigido, ante todo, a facilitar la instalación de grandes empresas, con frecuencia maquiladoras, y a proporcionar la infraestructura necesaria para tal efecto.

d) En los dos estados, el sector tal vez más golpeado por la crisis reciente ha sido el agropecuario, lo cual ha influido en la multiplicación del desempleo y, más aún, del subempleo o del sector informal.

e) Investigaciones de campo realizadas en años recientes por María de los Angeles Crummet en Aguascalientes y por el que esto escribe en Tlaxcala (nota 8) confirman la reactivación de una doble tendencia para lograr la sobrevivencia en las dos entidades: por una parte, el nuevo auge de la maquila doméstica en amplias zonas de ambos estados y, por otra parte, el incremento de la emigración aunque en direcciones opuestas. En Aguascalientes, la dirección predominante de los emigrantes rurales apunta hacia los Estados Unidos, mientras que en Tlaxcala predomina la emigración hacia el Distrito Federal, bien sea en forma más o menos definitiva o según un modelo oscilatorio de frecuencia casi diaria.

Todas estas "bondades" son aprovechadas por el gobierno regional y por los empresarios, sean locales o internacionales, para instalar una serie de empresas de avanzada tecnología y generadoras de insuficientes fuentes de trabajo. Ello es posible porque en ambos estados cuentan con las condiciones adecuadas para la instalación de industrias modernas y, además, en ellos existen otros factores -como la emigración, la maquila domiciliaria casi siempre clandestina, etc.- que actúan como colchones contra la crisis rampante.

los países miembros de la Comunidad Económica Europea durante la década de los ochenta.

7. Posteriormente especificaremos las características de estas nuevas oleadas tal como aparecen en las empresas que están llegando al estado de Tlaxcala.

8. Los datos sobre el caso de Aguascalientes se encuentran en el ya citado libro de Javier Delgadillo (107 - 125). Para el estado de Tlaxcala tomamos como punto de referencia el artículo: Alonso, José A. (1997), "Efectos del TLCNA en la microindustria del vestido de Tlaxcala, México". En: "Comercio Exterior", vol. 47, n.2 (febrero), 103 - 110. Otros estudios complementarios aparecen en : Alonso, José A. (1991): "La industria domiciliaria del vestido en Tlaxcala. Una investigación exploratoria": En Acta Sociológica, vol. IV, n. 1 (enero - abril), 47 - 61; Creummet, María de los Ángeles (1998): "Procesos de cambio en las funciones de clase y género después de una década de austeridad. Hogares rurales en Aguascalientes, México". En Florencia Peña y José A. Alonso (edit.) : Estrategias Femeninas Para Enfrentar La pobreza. el trabajo domiciliario en la elaboración De prendas de vestir, México: Escuela Nacional de Antropología e Historia y Universidad Autónoma de Tlaxcala (en prensa). 
Por otra parte, en ambos estados se observan fundamentales divergencias en cuanto a los procesos de industrialización promovidos en los últimos años. Aguascalientes es quizás el estado mexicano que más ha cambiado su fisonomía industrial debido a la instalación masiva de industrias de punta de las ramas electrónica, automotriz y metalmecánica; mientras que las industrias tradicionales textiles y del vestido han perdido fuerza. La tendencia opuesta parece predominar en el estado de Tlaxcala. Aunque un buen número de industrias modernas (químicas, metalmecánicas,etc.) se han instalado en diversas zonas del estado, el TLCNA parece haber dado la señal de entrada al capital norteamericano para invertir sobre todo en la rama del vestido a través de una novedosa red de empresas maquiladoras de nivel internacional (Alonso, 1997).

Esta observación nos conduce a otra discrepancia importante entre los dos estados. Aunque tanto en Tlaxcala como en Aguascalientes se observa un recrudecimiento de la maquila, incluso domiciliaria, resulta decisivo subrayar las diferencias existentes en el proceso de 'maquilización' a nivel regional. A falta de ulterior información, todo parece indicar que en Aguascalientes -y, en general, en El Bajío- las nuevas maquilas responden a necesidades más bien regionales o nacionales. En consecuencia, las redes productivas que siempre se desarrollan alrededor de las maquilas son controladas y diseñadas por empresarios regionales.

En el caso de Tlaxcala, la situación es radicalmente diferente. Mientras el factor nacional pierde vigor, el capital norteamericano crece y se extiende en casi todo el estado, apoyándose directamente en medianas y pequeñas empresas. Este rasgo parece ser la clave para entender a las NUEVAS REDES que están apareciendo en Tlaxcala a partir de 1996". $\mathrm{El}$ aspecto novedoso es que muchos medianos y pequeños empresarios tlaxcaltecas se convierten en exportadores, como maquiladores y a través de intermediarios o "brokers" de nacionalidad norteamericana, pero que instalan sus bodegas en territorio nacional. El precio que deben pagar estos fabricantes mexicanos por convertirse en exportadores es renunciar a ser productores autónomos e independientes. Los promotores a ultranza del TLCNA podrían hasta proclamar que ahora en México ya no exportan solamente las grandes empresas; también los pequeños y medianos empresarios en números crecientes son capaces de exportar.

\section{CONCLUSIÓN}

Todo hace sospechar que Tlaxcala no será el único estado que se integre a estas nuevas redes maquiladoras. De hecho, las bodegas construidas por los "brokers" o intermediarios ya están distribuidas en diversos estados de la república. Nos hallamos, según parece, ante una nueva clase de maquiladoras que nos obligan a enriquecer la tipología propuesta por (Gereffy: 1993, 239 - 271). Este autor menciona dos tipos de maquiladoras: las 'viejas' maquiladoras que requieren mano de obra intensiva y, por tanto, combinan los salarios minúsculos con el trabajo a destajo de las mujeres. Predominan estas maquiladoras en las manufacturas ligeras y hasta ahora estaban en manos casi siempre norteamerica- nas. Las 'nuevas' maquiladoras, por el contrario, son en parte japonesas -las de Aguascalientes, por ejemplo- y emplean considerables inversiones en alta tecnología, así como fuerza de trabajo masculina.

Frente a estos dos tipos 'clásicos' de la industria maquiladora mexicana, el nuevo tipo observado en Tlaxcala representa un producto genuino del TLCNA. Es difícil que los japoneses y coreanos puedan desarrollar redes parecidas. Se requiere la proximidad geográfica para mantener activas y bien coordinadas redes tan complejas. De hecho, ya existen quejas por parte de los empresarios mexicanos con respecto a los 'brokers' o intermediarios yanquis por no cumplir adecuadamente las obligaciones contraidas. En este sentido el papel de estas 'novísimas' maquiladoras parece crucial para fomentar la "integración" (?) de las tres naciones norteamericanas; es decir, para estrechar los vínculos de subordinación -que no de simple cooperación-entre los componentes del bloque norteamericano.

No es de extrañar que ya en 1995 la ex negociadora Carla Hills estimara que el TLCNA representa la garantía de que México seguirá en el camino económico correcto después de la crisis de 1994 y de que no aplicará, dijo, las medidas 'equivocadas' que se adoptaron en $1982^{10}$.

Tampoco faltan voces mexicanas que manifiestan opiniones opuestas. El empresario Daniel Ríos " opinaba unos meses después que muchos negocios mexicanos han dejado de operar debido "a la competencia desmesurada por parte de empresarios estadounidenses y canadienses, que prefirieron establecer sus compañías en este país". En referencia a las industrias mexicanas señaló que "la mayoría de las firmas mexicanas productoras han tenido un cambio de estrategia, pues sus plantas productivas las convierten ahora en centros de distribución que representan mayores márgenes de utilidad". Nos hallaríamos, por tanto, frente a una nueva posibilidad para 'colaborar' con el capital norteamericano. En este caso la planta productiva ha dejado de serlo para convertirse en comercializadora.

En definitiva, son las dos caras del espejo que nos ha traído el Tratado de Libre Comercio de Norte América.

\section{BIBLIOGRAFÍA}

AlONSO, José A. (1991): "La industria domiciliaria del vestido en Tlaxcala. Una investigación exploratoria”, Acta Sociológica, vol. IV, n. 1: 47 - 61 (enero - abril).

- (1997): "Efectos del TLCNA en la microindustria del vestido de Tlaxcala, México". Comercio Exterior, vol. 47, n.2: 103 - 110 (febrero).

ARRIOLA, Gabriela (1997): La nueva ola proteccionista norteamericana. Tesis de licenciatura presentada en el Departamento de Relaciones Internacionales de la Universidad de las Américas - Puebla.

9. Este proceso aparece largamente comentado en el artículo de 1997 publicado en Comercio Exterior (ver la nota 8). Aquí sólo mencionamos los aspectos más relevantes. 1995.

10. El Financiero, diario de la Ciudad de México, 31 de octubre de

11. El Financiero, diario de la Ciudad de México, 22 de mayo de 1996. 
Brown, Timothy C. (1997): "The Fourth Member of NAFTA: The U.S. - Mexico Border". En The Annals Of The American Academy Of Political And Social Science, vol. 550: 105 - 129 (march).

Cordera, Rolando y Carlos Tello (1981): México: la disputa por la Nación. México: Editorial Siglo XXI.

Creummet, María de los Ángeles (1998): "Procesos de cambio en las funciones de clase y género después de una década de austeridad. Hogares rurales en Aguascalientes, México”. En Peña, Florencia y Alonso, José A. (edit.): Estrategias femeninas para enfrentar la Pobreza. El trabajo domiciliario en la elaboración de prendas de vestir, México: Escuela Nacional de Antropología e Historia y Universidad Autónoma de Tlaxcala.

Delgadillo Macías, Javier (1993): El Desarrollo regional de México ante los nuevos bloques económicos. México: Instituto de Investigaciones Económicas de la Universidad Nacional Autónoma de México.

Díaz CAYEROS, Alberto (1995): Desarrollo económico e inequidad regional: hacia un nuevo pacto federal en México. México, Distrito Federal: Miguel Angel Porrua editor.

Estay Reyno, Jaime (1996): Pasado y Presente de la Deuda externa de América Latina. México: Instituto de Investigaciones Económicas de la Universidad Nacional Autónoma de México y Universidad Autónoma de Puebla.

Fossaert, Robert (1991): Le Monde au 21e. Siecle. Paría: Fayard.
GerEFFI, Gary (1993): “Cómo contribuyen las industrias maquiladoras al desarrollo nacional de México y a la integración de América del Norte?”. En: Gustavo Vega Cánovas (coordinador): Liberación económica y Libre Comercio en América del Norte. México: El Colegio de México.

Gutiérrez Haces, María Teresa (1992): “La Negociación del TLC a partir del borrador de Dallas”. En Problemas Del Desarrollo, vol. XXIII, n. 90: 66 - 71 (julio - septiembre).

MORALES, Isidro (1996): "Hierarchical and arms length markets under NAFTA. The case of Mexican petrochemical and textile industries" Versión mimeografiada.

SAXE-FERnÁndEZ, John (1992): “América Latina- Estados Unidos en la posguerra fría: apuntes estratégicos preliminares”, Problemas Del Desarrollo, vol. XXIII, 90: 135 - 179 (julio - septiembre)

Talavera Déniz, Pedro y Martínez Peinado, Javier (1995): "La Estructura Económica Mundial: El Comercio Centro-Periferia”. Barcelona: McGraw Hill.

VArios Autores (1990): América Latina: Critica del Neoliberalismo. México, D.F.: Centro de Estudios para un Proyecto Nacional Alternativo.

VilaseCA, Jordi y REQuena (1995): "Las Instituciones de Integración Económica”. En: Economía Mundial, editado por Javier Peinado et al., Barcelona: McGraw Hill.

WALlERSTEIN, Immanuel (1979): El Moderno sistema mundial, dos volúmenes, México: Siglo XXI editores (primera edición en español).

\section{RESUMEN}

La apertura de la economía mexicana se incrementó a partir de 1985, cuando el gobierno mexicano adoptó la estrategia neoliberal. Esta política se profundizó aún más en 1994 al entrar en vigor el Tratado de Libre Comercio de Norteamérica (en adelante, TLCNA). Desde entonces el país más beneficiado ha sido los Estados Unidos, para los que el TLCNA no es sino un paso decisivo en la construcción del 'bloque americano'. A nivel nacional también se han agravado las desigualdades regionales de acuerdo con el grado y la forma de penetración de las empresas transnacionales (ETs). En el artículo se analizan los impactos de la apertura neoliberal en dos estados, Aguascalientes y Tlaxcala, para poder comprender las diferencias que ya se observan en ambas entidades. En esta primera etapa, la principal diferencia -según parece- radica en las NUEVAS REDES DE SUBCONTRATACIÓN que proliferan en el estado de Tlaxcala bajo la dirección de las ETs. En definitiva, la apertura propiciada por el TLCNA favorece la creciente penetración del capital norteamericano en México y la polarización de la industria mexicana en dos sectores: uno, minoritario, asociado como subcontratista a las empresas transnacionales y otro, mayoritario, que difícilmente puede sobrevivir y que cada vez más recurre a la 'informalidad' (=clandestinidad) para evitar su desaparición.

Palabras clave: México, Estados Unidos, Tratado de Libre Comercio de Norteamérica, dependencia.

\section{ABSTRACT}

The Mexican government opened the doors to foreign capital in 1985, when the president Miguel de la Madrid adopted the neoliberal strategy. Later this openness led president Carlos Salinas de Gortari to the adoption of the NORTH AMERICAN FREE TRADE AGREEMENT (NAFTA) as the best way to promote the welfare of the Mexican people. While the USA has been the largest beneficiary of this agreement, conceived by American politicians as a useful step to counterbalance the power of Japan and the European community, Mexico has received the full impact of this new strategy in terms of the increasing inequalities brought about by foreign firms - mostly American- in the Mexican industrial scene. This article examines the NAFTA impact on the Mexican economy by comparing some of the changes that seem to be present in two states, namely Aguascalientes and Tlaxcala in recent years. Both states have received large amounts of foreign investment, but it seems that American brokers have been able to set up a complicated network of 'maquiladoras' (assembling industries) in the state of Tlaxcala which is missing in Aguascalientes. Unlike the state of Aguascalientes, the last result of this American penetration in the state of Tlaxcala is the assimilation of the largest Mexican enterprises within the American-led networks and, consequently, the inability of most small and micro Tlaxcalan industries to survive. Their only hope lies in the informal economy.

Key Words: Mexico, United States, NAFTA, dependence. 


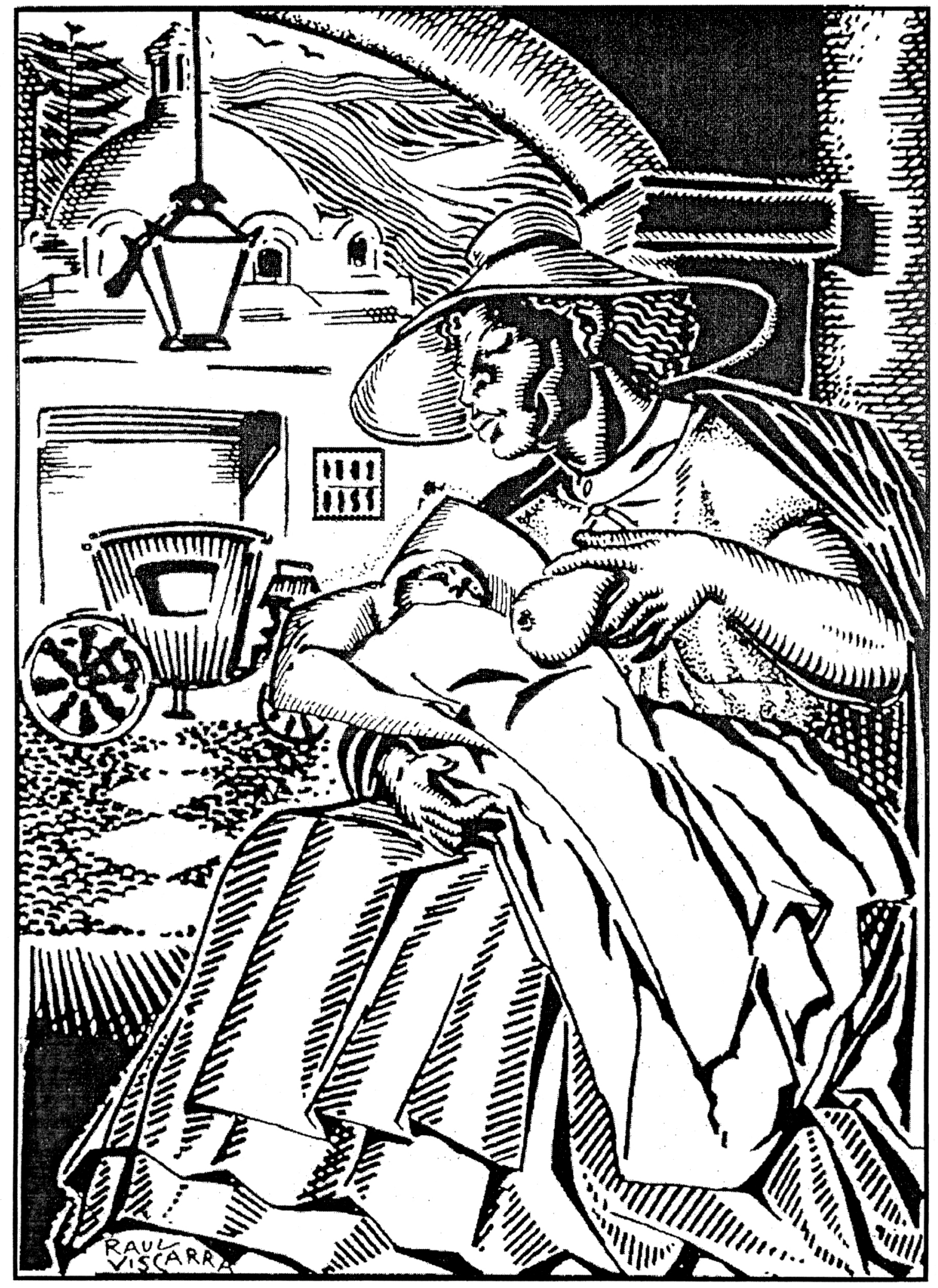

